
\title{
R Reserach S Suare \\ Performance Analysis of Vertically Stacked Nanosheet Tunnel Field Effect Transistor with Ideal Subthreshold Swing
}

Garima Jain ( $\sim$ garima.bansal0390@gmail.com )

Guru Nanak Dev University

Ravinder Singh Sawhney

Guru Nanak Dev University

Ravinder Kumar

Guru Nanak Dev University

Amit Saini

Uttar Pradesh Technical University: Dr A P J Abdul Kalam Technical University

\section{Research Article}

Keywords: Nanosheet-Tunnel Field Effect Transistors (NS-TFET), Nanosheet-Field Effect Transistors (NSFET), Short Channel Effects (SCE), Band to Band Tunneling (BTBT), Subthreshold Swing (SW)

Posted Date: April 26th, 2021

DOl: https://doi.org/10.21203/rs.3.rs-429581/v1

License: (9) This work is licensed under a Creative Commons Attribution 4.0 International License. Read Full License

Version of Record: A version of this preprint was published at Silicon on August 5th, 2021. See the published version at https://doi.org/10.1007/s12633-021-01302-1. 


\section{Abstract}

In this paper, a novel vertically stacked silicon Nanosheet Tunnel Field Effect Transistor (NS-TFET) device scaled to a gate length of $12 \mathrm{~nm}$ with Contact poly pitch (CPP) of $48 \mathrm{~nm}$ is simulated. NS-TFET device is investigated for its electrostatics characteristics using technology computer-aided design (TCAD) simulator. The inter-band tunneling mechanism with a P-I-N layout has been incorporated in the stacked nanosheet devices. The asymmetric design technique for doping has been used for optimum results. NSTFET provides a low leakage current of order $10^{-16} \mathrm{~A}$, an excellent subthreshold swing (SW) of $23 \mathrm{mv} /$ decade, and negligible drain induced barrier lowering (DIBL) having a value of $10.5 \mathrm{mv} / \mathrm{V}$. The notable ON to OFF current ratio of the order of $10^{11}$ has been achieved. The device exhibits a high transconductance of $3.022 \times 10^{-5} \mathrm{~S}$ at the gate to source voltage of 1V. NS-TFET shows tremendous improvement in short channel effects (SCE) and is a good option for advanced technologies.

\section{Introduction}

Nanosheets have emerged as a potential successor to conventional Finfets and stacked nanowires for $7 \mathrm{~nm}$ technology and beyond [1-3]. Finfets need tall and thin fins, which enhance the fabrication cost and complexity [4] while the surface roughness factor degrades the performance of stacked nanowires [5]. The Nanosheet Field Effect Transistor (NS-FET) exhibits enormous current density due to its increased effective width per footprint [6]. NS-FET has good electrostatics control and hence remains immune to short channel effects [7]. It has been reported that NS-FET shows superior electrostatic performance in comparison to stacked nanowires or Finfets [8]. Thus NS-FET with 3D vertically stacked channels is a promising candidate for future advanced technology applications [6].

At low voltages, the device miniaturization below $50 \mathrm{~nm}$ leads to immense OFF-state power consumption and elevated subthreshold swing (SW) [9-10]. The SW at CPP of 48nm has been reported as $83 \mathrm{mv} /$ decade and $94 \mathrm{mv} /$ decade for nfet and pfet respectively [11].

The thermionic conduction mechanism of NS-FET makes SW temperature-dependent and this temperature constraint makes SW worse.

Vertically stacked Junctionless nanosheets(JL-Ns) show highly improved performance in terms of leakage current and SW [12]. JL-Ns with a different number of channels exhibit high ON current. At channel/oxide interface, JL-Ns provide indemnity for mobility degradation due to scattering of carriers [13]. The major bottleneck associated with JL-Ns is the exhibition of high leakage current while SW becomes non-scalable below $60 \mathrm{mv} /$ decade

Tunnel Field Effect Transistors (TFETS) provide a remedy for high OFF-state current and exhibit steep SW [14-16]. TFETs work on the principle of the BTBT mechanism. In NS-FETS, the BTBT mechanism can be incorporated; thus making it a novel device consisting of vertically stacked nanosheet tunnel field effect transistor (NS-TFET). Inner spacers can be treated as an underlapped region for TFETS. It has been further reported that underlapped source and drain regions assist in reducing ambipolarity [17-18]. The 
fabrication process of TFETs varies from that of Mosfets in terms of their source doping. Thus, the fabrication of NS-TFET is comparatively easier to implement with minimal deviation from the NS-FET fabrication process [19-20].

In Sect. 2, we have discussed the calibration characteristics of NS-FET. Section 3 describes the design of a vertically stacked NS-TFET device with three layers. In our proposed work, the design parameters of the NS-TFET device are optimized to achieve good electrostatics. To the best of our knowledge, no tunnelingbased Silicon stacked nanosheets with P-I-N configuration have been reported in the literature. Section 4 highlights the performance metrics of NS-TFET. The ultimate aim of NS-TFET is to tune the tunneling barrier at the extended source-channel junction; thereby reducing short channel effects such as low leakage current, minimal DIBL, and superior Subthreshold swing. Section 5 explains the drawn conclusions.

\section{Calibration Characteristics}

Vertically stacked Nanosheet transistors exhibit excellent ON-current density due to their increased effective width. The reference model of NS-FET with three layers having CPP of 48nm, the gate length of $12 \mathrm{~nm}$, and a sheet thickness of $5 \mathrm{~nm}$ are simulated on a visual TCAD platform [21]. The inner spacer thickness and the width of NS-FET are kept at $5 \mathrm{~nm}$ and 50nm respectively. The vertical sheet-to-sheet spacing is $10 \mathrm{~nm}$. A combination of high- $\mathrm{k}$ dielectric material $\mathrm{HFO}_{2}$ having thickness $1.28 \mathrm{~nm}$ and $\mathrm{SiO}_{2}$ having thickness of $0.5 \mathrm{~nm}$ has been used for effective oxide thickness of $0.7 \mathrm{~nm}$. The symmetrical source and drain doping have a value of $3 \times 10^{20} \mathrm{~cm}^{-3}$ while the channel doping is $1 \times 10^{17} \mathrm{~cm}^{-3}$. Titanium Nitride material has been selected for gate with gate metal work function of $4.7 \mathrm{eV}$. The calibration graph of the simulation result is benchmarked with the experimental data at a drain voltage of $0.65 \mathrm{~V}$ as shown in Fig. 1.

\section{Device Structure}

The dimensions of NS-TFET are in accordance with the reference structure [21]. The proposed NS-TFET device differs from the NS-FET device in terms of the type of doping. The reference structure has n-type symmetrical doping in both source and drain [21] while in NS-TFET; asymmetrical P-I-N configuration has been employed. P-I-N design with BTBT mechanism shows tremendous improvement in short channel effects (SCE). In the BTBT mechanism, the width of the tunneling barrier is modulated on the variation of gate voltage at constant drain voltage. The asymmetric lower drain doping is used to curb ambipolar behavior [22-23]. Work function engineering has been done to achieve desirable results. For simulation of NS-TFET, the work function is chosen to be $5.00 \mathrm{eV}$. Figure 2a represents the 3-D view of NS-TFET. Figure $2 \mathrm{~b}$ depicts a cross-sectional view of NS-TFET with P-I-N configuration. The geometric parameters, doping concentrations of NS-TFET are listed in Table 1.

Physical models used in Genius code define the behavior of semiconductor devices [22]. These models specify physical parameters like mobility, recombination rate, etc. Drift-Diffusion (DD) model is the 
fundamental solver for Poisson's and continuity equations [23] and has been recommended to use for determining the transport of charge carriers and computation of the drain current. The Lombardi model is invoked for carrier mobility in the inversion layer of the NS-TFET device. This mobility model incorporates bulk mobility, mobility due to surface charge, and scattering [24]. Gate tunneling plays a pertinent role in NS-TFET devices. Kane's Model invokes the BTBT mechanism for carrier generation. For 3D simulations; Kane's model provides better convergence results [31]. Shockley-Read-Hall (SRH) model has been considered for carrier recombination mechanism and it stimulates the leakage current that determines loff in TFETs [32].

Table 1

The design parameters of vertically stacked NS-TFET

\begin{tabular}{|ll|}
\hline Parameters & Dimension \\
\hline Gate length $\left(L_{g}\right)$ & $12 \mathrm{~nm}$ \\
\hline Gate work function of n-NS-TFET & $5.0 \mathrm{eV}$ \\
\hline Gate work function of p-NS-TFET & $4.23 \mathrm{eV}$ \\
\hline Source Doping (Na) & $3 \times 10^{20} \mathrm{~cm}^{-3}$ \\
\hline Drain Doping, (Nd) & $1 \times 10^{17} \mathrm{~cm}^{-3}$ \\
\hline Channel Doping, (Nch) & $1 \times 10^{16} \mathrm{~cm}^{-3}$ \\
\hline Effective oxide thickness, (EOT) & $0.7 \mathrm{~nm}$ \\
\hline Nanosheet width, (Ns_W) & $50 \mathrm{~nm}$ \\
\hline Nanosheet Thickness, (Ns_Th) & $5 \mathrm{~nm}$ \\
\hline
\end{tabular}

\section{Results And Discussion}

Three-dimensional simulations of NS-TFET are done using COGENDA-TCAD software [24]. The physical models such as the DD model, Lombardi mobility model, Kane's BTBT model, and SRH model are evaluated at each mesh node using TCAD software. The performance metrics of vertically stacked NSTFET device has been discussed in this section.

\subsection{Triple nanosheet}

Gate all-around devices provide little room for carriers to drift when the transistor is in ON state [25]. The stacking of nanowires increases the effective width and allows the carriers to flow, but the increased device capacitance along with surface roughness decreases the speed of carriers [26]. Stacking thin nanosheets atop one another enhances the effective width and hence provides larger room for carriers to 
flow. This further enables the large drive current while maintaining constricted control of the leakage current [25-26].

We have performed the simulations for vertically stacked single, double and triple NS-TFETs at the drain to source voltage $(\mathrm{Vds})$ of $0.65 \mathrm{~V}$. The plot of drain current of single nanosheet (Id_1ns), double nanosheet (Id_2ns), and triple nanosheet (Id_3ns) is depicted in Fig. 3. The results show that ON-current increases exponentially in the case of double and triple NS-TFETs as compared to single NS-TFET. The drain current of Id_3ns and Id_2ns is observed to be 2.0168 and 3.03 times higher than Id_1ns respectively. Ion/loff ratio in triple-stacked NS-TFET is 9.557 times that of single NS-TFET while with double-stacked NS-TFET, it is observed to be 4.73 times high. Hence, three-layered NS-TFET exhibits superior performance in terms of drive current

\subsection{Energy Band Diagram}

The NS-TFET exhibits similar behavior to that of n-TFET on the application of constant drain voltage. In NS-TFET, an interband tunneling conduction mechanism has been incorporated. In the OFF state of the ptype extended source of NS-TFET, very few electrons are available at the conduction band of the source for injection into the channel. This results in negligible movement of carriers and hence poor leakage current. With variation in the gate voltage, the energy band of the channel varies relative to the extended source. From Fig. 4 , it is evident that at saturation voltage of $\mathrm{Vds}=0.65 \mathrm{~V}$ with positive gate to source voltage $(\mathrm{Vgs}>0 \mathrm{~V})$, the valence band of the extended source is aligned with the conduction band of the channel. The carriers tunnel through the potential barrier between the valence band of the extended source and the conduction band of the channel. These charge carriers present in channel drift towards the extended drain (ext_d) to produce drain current.

\subsection{On Current and OFF current}

Figure 5 depicts the transfer characteristics of NS-TFET. In the off state of TFETs with zero gate to source voltage, the movement of charge carriers from the valence band of the extended source region into the channel region is hindered due to large tunneling barrier width. Hence, the leakage current is extremely low. In the off state, linear leakage current(I_Lin) is of the order $10^{-16} \mathrm{~A}$ at gate voltage $\mathrm{Vgs}=0 \mathrm{~V}$ with drain voltage $\mathrm{Vds}=0.10 \mathrm{~V}$. On application of positive gate voltage, bandgap modulation takes place as shown in Fig. 4. With the gradual increase in gate bias, the bands of the channel are lowered; thus enabling more electrons to tunnel from the valence band of the extended source into the conduction band of the channel. The tunneling barrier width is reduced near the extended-source channel region leading to a steep increase in the drain current. At saturation voltage of $\mathrm{Vds}=0.65 \mathrm{~V}$, leakage current is of order $10^{-14} \mathrm{~A}$. The saturation $\mathrm{ON}$ current (I_sat) reported is $1.26 \times 10^{-5} \mathrm{~A}$ at $\mathrm{Vds}=0.65 \mathrm{~V}$ with $\mathrm{Vgs}=1.2 \mathrm{~V}$. Thus, the lon/loff ratio for three-layered NS-TFET is $1.101 \times 10^{11}$. This high lon/loff ratio is desirable for high-performance nanoscale devices. 
Table 2

Figures of merit of NS-TFET

\begin{tabular}{|lll|}
\hline Figures of Merit & Values of n-NS-TFET & Values of p-NS-TFET \\
\hline Leakage current & $1.0144 \times 10^{-16} \mathrm{~A}$ & $1.16147 \times 10^{-16} \mathrm{~A}$ \\
\hline Ion/loff ratio & $1.101 \times 10^{11}$ & $9.378 \times 10^{11}$ \\
\hline Threshold Voltage & $0.402 \mathrm{~V}$ & $0.400680 \mathrm{~V}$ \\
\hline DIBL & 10.5 & 11.4 \\
\hline Subthreshold swing & $23 \mathrm{mv} /$ decade & $23.786 \mathrm{mv} /$ decade \\
\hline
\end{tabular}

\subsection{Sub-threshold swing and DIBL}

The main premise of designing NS-TFET as an alternative to NS-FET is due to its refined subthreshold swing. NS-FETs operate on a thermionic injection mechanism and thus have a thermal limit of $60 \mathrm{mv} /$ decade [27]. In NS-TFETs, the BTBT conduction mechanism is utilized. NS-TFET offers desired steep SW of $23 \mathrm{mv} /$ decade at low Vds $=0.10 \mathrm{~V}$ with the desired ON-state performance. For this subthreshold regime, the threshold voltage is $0.402 \mathrm{~V}$ and negligible Drain induced barrier lowering (DIBL) of 10.5 is found. SW gives a higher lon/loff ratio and thus makes itself apt for faster switching circuitry. Table 2 represents the performance metrics of NS-TFET in terms of threshold voltage, SW, DIBL, etc.

\subsection{Ambipolairty}

The transfer characteristics of three-layered NS-TFET under different doping concentrations have been represented in Fig. 6 . It is evident from the figure that NS-TFET shows its ambipolar behavior when it is subjected to the negative gate to source voltage $(\mathrm{Vgs}<0 \mathrm{~V})$. The electrons tunnel from the channel to the conduction band of the extended drain and thus results in the current flow of the same polarity and hence behave as p-type. This behavior is not desirable in digital circuitry where tunneling between channel and drain is curbed [28-30]. The best results are observed for the doping concentration of $10^{17} \mathrm{~cm}^{-3}$. It is evident that with the decrease in the doping concentration of drain, an ambipolar current is reduced up to a considerable amount. The depletion width of the drain side increases due to lower drain doping concentration. As a result, ambipolar current reduces.

\subsection{Transconductance $\left(\mathrm{g}_{\mathrm{m}}\right)$,Transconductance generation efficiency(TGF) and Total Gate Capacitance $\left(\mathrm{C}_{\mathrm{gg}}\right)$}

Transconductance $\left(g_{m}\right)$ is a performance metric, which reflects the device efficiency in terms of effective input voltage conversion into output current [31]. It is described by first-order differentiation of drain current with reference to the gate to source voltage [32]. 


$$
g_{m}=\left.\frac{\partial I_{d d}}{\partial V_{G S}}\right|_{I_{D S}}
$$

where $I_{d d}$ represents current tunneling from the source terminal to the drain end. $V_{g s}, V_{d s}$ represents the gate to source voltage and constant drain voltage respectively.

Transconductance generation efficiency (TGF) is another vital parameter that determines the efficiency of NS-TFET in terms of conversion of the current into transconductance $\left(g_{m}\right)$ and is given by [33].

$$
T G F=\frac{g_{m}}{I_{d d}} \mathrm{~V}^{-1}
$$

Figure $7 \mathrm{a}$ and $7 \mathrm{~b}$ represent the variation in transconductance and TGF with respect to the gate-source voltage respectively. It is unambiguously clear that NS-TFET exhibits comparatively higher transconductance due to the hike in tunneling of carriers in the channel. For the selected value of $I_{d d}=10^{-}$ ${ }^{10} \mathrm{~A}, \mathrm{TGF}$ is found to be $54 \mathrm{~V}^{-1}$.

The plot of the variation of total gate capacitance $\left(C_{g g}\right)$ with respect to $\mathrm{Vgs}$ is depicted in Fig. $7 \mathrm{c} . C_{g g}$ at $V g s=1 \mathrm{~V}$ is found to be $1.662 \times 10^{-17} \mathrm{~F}$.

\section{p-Ns-TFET design:}

The vertically stacked NS-TFET with N-I-P configuration has been designed to demonstrate its p-type characteristics. The geometry parameters are kept the same as mentioned in Table 1. Work function engineering has been implemented to match $\mathrm{ON}$ and leakage currents. For p-SN-TFET, the work function is kept at $4.23 \mathrm{eV}$. The source has donor impurities with a concentration of $3 \times 10^{20} \mathrm{~cm}^{-3}$ while the drain has acceptor impurities having a concentration of $10^{17} \mathrm{~cm}^{-3}$. The transfer characteristics of $p$-SN-TFET at linear voltage $\mathrm{Vds}=-0.10 \mathrm{~V}$ and saturation voltage of $\mathrm{Vds}=-0.65 \mathrm{~V}$ are simulated using TCAD. The simulation results of both $\mathrm{p}$ and $\mathrm{n}$-type at linear and saturation voltage are displayed in Fig. 8 . The performance metrics of p-NS-TFET are mentioned in Table 2.

\section{Conclusion}

In this paper, vertically stacked NS-TFET with three layers has been modeled and simulated. The short channel effects have reduced tremendously by using the BTBT mechanism with P-I-N configuration. A high lon/loff ratio with a low leakage current has been achieved. The steep subthreshold swing of 23 $\mathrm{mv} /$ decade with negligible DIBL makes NS-TFET useful for low power applications. High Transconductance, device efficiency, total capacitance parameters of NS-TFET have been extracted. $p$ TFET configuration for NS-TFET has also been proposed which makes it apt for faster switching 
applications. All these advantages make NS-TFET a viable option for next-generation applications and towards future advancements.

\section{Declarations}

Funding Not applicable

Conflicts of interest/Competing interests the authors have declared that no competing interests exist.

Availability of data and material Not applicable

Code availability Not applicable

Compliance with Ethical Standards This study was ap-proved by the university research ethics committee. All procedures per-formed in this study follow the ethical standards of the institutional and research committee.

\section{Consent for publication Yes}

Authors' contributions All authors contributed to the design and simulation. Material preparation, data collection and analysis were performed by Garima Jain, Dr. Ravinder Singh Sawhney, Dr. Ravinder Kumar and Amit Saini. The first draft of the manuscript was written by Garima Jain and all authors commented on previous versions of the manuscript. All authors read and approved the final manuscript.

\section{Acknowledgements}

We thank the Group, department of Electronics Technology, Guru Nanak Dev University, Amritsar for their interest in this work and useful comments to draft the final form of the paper. The support of CADRE Design Systems is gratefully acknowledged. We would like to thank Guru Nanak Dev University, Amritsar and Cadre Design Systems for lab facilities and research environment to carry out this work.

\section{References}

1. D. Jang, D. Yakimets, G. Eneman, P. Schuddinck, and M. G. Bardo, "Device Exploration of NanoSheet Transistors for Sub-7-nm Technology Node," IEEE Trans. Electron Devices, vol. 64, no. 6, p. pp. 27072713, June 2017. DOI: 10.1109/TED.2017.2695455

2. S. Barraud, V. Lapras, B. Previtali, M. P. Samson, J. Lacord, S. Martinie, M. A. Jaud, S. Athanasiou, F. Triozon, O. Rozeau, J. M. Hartmann, C. Vizioz, C. Comboroure, F. Andrieu, J. C. Barbé, M. Vinet and T. Ernst, "Performance and Design Considerations for Gate-All-Around Stacked-NanoWires FETs," IEEE International Electron Devices Meeting (IEDM), 2017. DOI: 10.1109/IEDM.2017.8268473

3. D. Yakimets, M. G. Bardon, D. Jang, P. Schuddinck, Y. Sherazi, P. Weckx, K. Miyaguchi, B. Parvais, P. Raghavan, A. Spessot, D. Verkest, and A. Mocuta, "Power-aware FinFET and lateral nanosheet FET targeting for 3nm CMOS technology," in IEEE International Electron Devices Meeting (IEDM), San 
Francisco, CA, USA, 2017.

DOI: 10.1109/IEDM.2017.8268429

4. X. He, J. Fronheiser, P. Zhao, Z. Hu, S. Uppal, X. Wu, Y. Hu, R. Sporer, L. Qin, R. Krishnan, E. M. Bazizi, R. Carter, K. Tabakman, A. K. Jha, H. Yu, O. Hu, D. Choi, J. G. Lee, S. B. Samavedam and D. K. Sohn, "Impact of aggressive fin width scaling on finfet device characteristics," in IEEE International Electron Devices Meeting (IEDM), San Francisco, CA, USA, 2017. DOI: 10.1109/IEDM.2017.8268427

5. F. M. Bufler, R. Ritzenthaler, H. Mertens, G. Eneman, A. Mocut and N. Horiguchi, "Performance Comparison of n-Type Si Nanosheets, and FinFETs by MC Device Simulation," IEEE Electron Device Lett., vol. 39, no. 11, p. 1628-1631, Nov 2018. DOI: 10.1109/LED.2018.2868379

6. P. Feng, S. Song, G. Nallapati, J. Zhu, J. Bao, V. Moroz, M. Choi, X. Lin, Q. Lu, B. Colombeau, N. Breil, M. Chudzik and C. Chidambaram, "Comparative Analysis of Semiconductor Device Architectures for 5-nm Node and Beyond pp.," IEEE Electron Device Lett., vol. 38, no. 12, p. 1657-1660, Dec 2017.DOI: 10.1109/LED.2017.2769058

7. K. Kalna, D. Nagy, A. J. García-Loureiro and N. Seoane, "3D Schrödinger Equation Quantum Corrected Monte Carlo and Drift Diffusion Simulations of Stacked Nanosheet Gate-All-Around Transistor," in IWCN, Wien: Institute for Microelectronics, TU Wien, May,2019, pp. 33-35.

8. S. D. Kim, M. Guillorn, I. Lauer, P. Oldiges, T. Hook and M. H. Na, "Performance Trade-offs in FinFET and Gate-All-Around Device Architectures for 7nm-node and Beyond," in 2015 IEEE SOI-3DSubthreshold Microelectronics Technology Unified Conference (S3S), Rohnert Park, CA, USA, Oct. 2015. DOI: $10.1109 /$ S3S.2015.7333521

9. D. Yakimets, M. G. Bardon, D. Jang, P. Schuddinck, Y. Sherazi, P. Weckx, K. Miyaguchi, B. Parvais, P. Raghavan, ,. A. Spessot, D. Verkest and A. Mocuta, "'’Power aware FinFET and lateral nanosheet FET targeting for 3nm CMOS technology," IEEE International Electron Devices Meeting (IEDM), p. 501504, 2017. DOI: 10.1109/IEDM.2017.8268429

10. L. Cai, W. Chen, G. Du, X. Zhang and X. Liu, "Layout Design Correlated With Self-Heating Effect in Stacked Nanosheet Transistors," IEEE Trans. Electron Devices, vol. 65, no. 6, pp. 2647-2653, June,2018. DOI: 10.1109/TED.2018.2825498

11. N. Loubet, T. Hook, P. Montanini, C. W. Yeung and S. Kanakasab, "Stacked nanosheet gateallaround transistor to enable scaling beyond FinFET," in Symposium on VLSI Technology, T230-T231, 2017. DOI: 10.23919/VLSIT.2017.7998183

12. D. Moon, S. Choi, J. P. Duarte and Y. Choi, "Investigation of Silicon Nanowire Gate-All-Around Junctionless Transistors Built on a Bulk Substrate," IEEE Transactions on Electron Devices, vol. 60, pp. 1355-1360, 2013. DOI: 10.1109/TED.2013.2247763

13. H. Chen, Y. Wu, C. Chang, M. Han, N. Lu and Y. Ch, "Performance of GAA Poly-Si nanosheet (2nm) channel of junctionless transistors with ideal subthreshold slope," in Symposium on VLSI Technology, 2013.

14. W. Y. Choi, B. Park, J. D. Lee and T. K. Liu, "Tunneling field-effect transistors (TFETs) with subthreshold swing (SS) less than $60 \mathrm{mV} /$ dec.," IEEE Electron Device Lett., vol. 28, no. 8, pp. 743-745, 
2007.DOI: 10.1109/LED.2007.901273

15. J. Colinge, FinFETs and other multi-gate transistors, New York: Springer, 2008. DOI: 10.1007/978-0387-71752-4

16. S. Kumar, E. Goel, K. Singh, B. Singh, P. K. Singh and K. Baral, "2-D analytical modeling of the electrical characteristics of dual-material double-gate TFETS with a sio2/hfo2 stacked gate-oxide structure.," IEEE Trans Electron Devices , vol. 64, no. 3, p. 960-968, 2017.

DOI:10.1109/TED.2017.2656630

17. R. Vaddi, R. P. Agarwal and S. Dasgupta, "Analytical modeling of subthreshold current and subthreshold swing of an underlap DGMOSFET with tied-independent gate and symmetricasymmetric options," J Microelectron, vol. 42, no. 5, pp. 798-807, 2011.

https://doi.org/10.1016/j.mejo.2011.01.004

18. G. Wadhwa and B. Raj, "Label Free Detection of Biomolecules Using Charge-Plasma-Based Gate Underlap Dielectric Modulated Junctionless TFET," Journal of Electronics Material, Springer, vol. 47, no. 8, pp. 4883-4893, May 2018. DOI: 10.1007/s11664-018-6343-1

19. W. Cao, D. Sarkar and Y. Khatami, "Subthreshold-swing physics of tunnel field-effect transistors," AIP, vol. 4, no. 6, pp. 067141-1-067141-9, 2014. https://doi.org/10.1063/1.4881979

20. V. Vijayvargiya, B. S. Reniwal, P. Singh and S. K. Vishvakarma, "Analogue/RF performance attributes of underlap tunnel field effect transistor for low power applications," Electron. Letters, vol. 52, no. 7, p. 559-560, 2016. DOI:10.1049/el.2015.3797

21. V. Jegadheesan, K. Sivasankaran and A. Konar, "Impact of geometrical parameters and substrate on analog/RF performance of stacked nanosheet field effect transistor.," Mater. Sci. Semicond. Process, vol. 93, pp. 188-195, 2019. DOI: 10.1016/j.mssp.2019.01.003

22. U. Dutta, M. K. Soni and M. Pattanaik, "Design and Optimisation of GATE-All-Around Tunnel FET for Low Power Applications," International Journal of Engineering \& Technology, vol. 7, no. 4, pp. 22632270, 2018.DOI: 10.14419/ijet.v7i4.12352

23. D. B. Abdi and M. J. Kumar, "Controlling Ambipolar Current in Tunneling FET's using Overlapping Gate-on Drain," Journal of the Electron Devices Society, vol. 2, no. 6, pp. 187-190, Nov. 2014.DOI: 10.1109/JEDS.2014.2327626 Genius Semiconductor Device Simulator Reference Manual

24. P. H. Vardhan, A. S. Ganguly and U. Gangu, "Threshold Voltage Variability in Nanosheet GAA Transistors," IEEE Trans. on Electron Devices, vol. 66, pp. 4433-4438, 2019. DOI:

10.1109/TED.2019.2933061

25. P. Ye, T. Ernst and M. V. Khare, "The last silicon transistor: Nanosheet devices could be the final evolutionary step for Moore's Law," IEEE Spectrum, vol. 56, 30 Jul 2019. DOI: 10.1109/MSPEC.2019.8784120

26. T. P. Dash, S. Dey, E. Mohapatra, S. Das and J. Jena, "Vertically-Stacked Silicon Nanosheet Field Effect Transistors at 3nm Technology Nodes," Devices for Integrated Circuit (DevIC), pp. 99-103, 2019. DOI: $10.1109 /$ DEVIC.2019.8783300 
27. H. Lu and A. Seabaugh, "Tunnel FET Transistors: State-of-the-Art," IEEE Journal of Electron Devices Society, vol. 2, no. 4, p. 44-49, June 2014. DOI: 10.1109/JEDS.2014.2326622

28. A. M. Ionescu and H. Riel, "Tunneling Field-Effect Transistors as Energy-Efficient Electronic Switches," Nature, vol. 479, pp. 329-337,, November 2011. DOI: 10.1038/nature10679

29. J. Kumar, R. Vishnoi and P. Pandey, Tunnel Field-Effect Transistors (TFET): Modelling and Simulation, John Wiley \& Sons, Ltd., 2017. DOI: 10.1002/9781119246312

30. S. Dash, G. S. Sahoo, and G. Prasad, "Improved cut-off frequency for cylindrical gate TFET using source delta doping," Procedia Technology, vol. 25, p. 450 - 455, 2016 . DOI:

10.1016/j.protcy.2016.08.131

31. U. Dutta, M. K. Soni and M. Pattanaik, "Simulation Study of Hetero Dielectric Tri Material Gate Tunnel FET based Common Source Amplifier Circuit," International Journal of Electronics \& Communications, vol. 9, pp. 2263-2270, 2018. DOI: 10.1016/j.aeue.2018.12.004

32. S.SS.M. Sze, Kwok K. Ng, "Physics of semiconductor devices," New Jersey, John Wiley \& Sons Inc. Publications, 2007, pp. 247-328. DOI:10.1002/0470068329

\section{Figures}

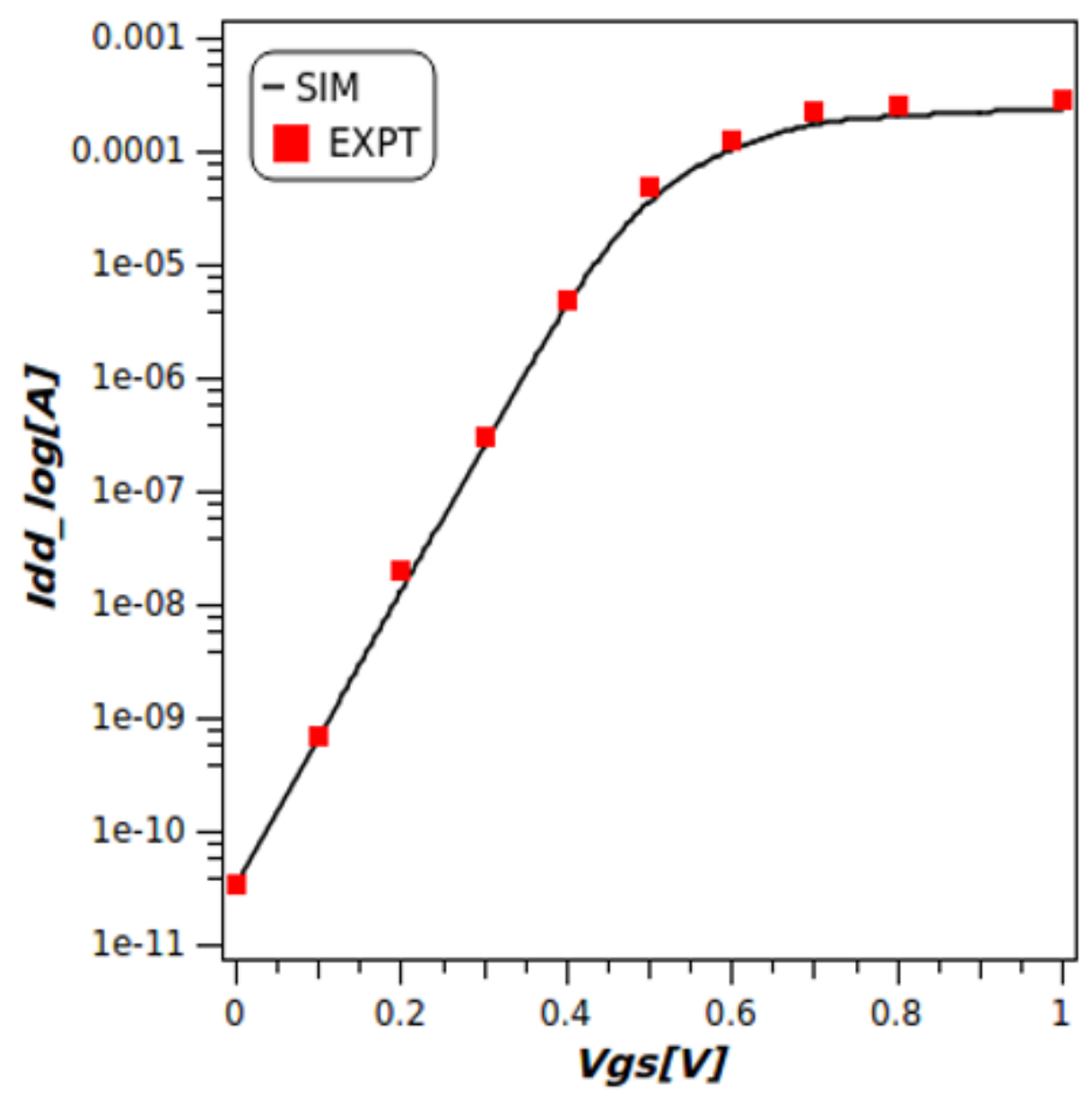

Figure 1 
Calibration graph of transfer characteristics of NS-FET [21]

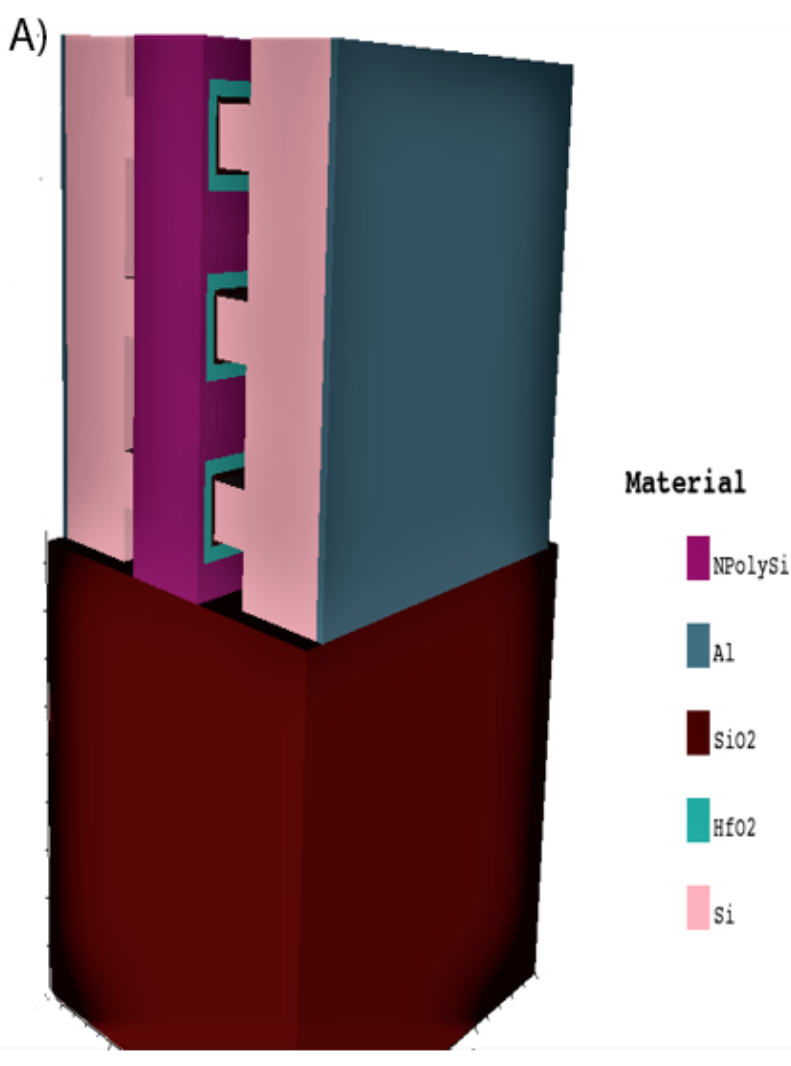

B)

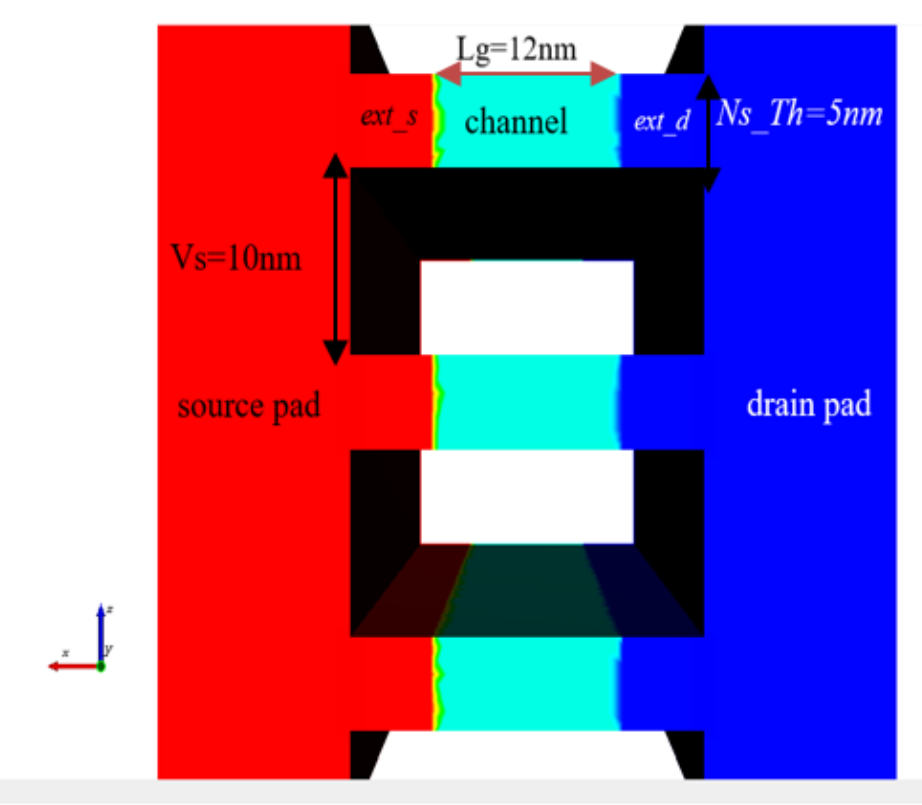

Figure 2

a: 3-D view of NS-TFET. b: Cross-sectional view of P-I-N tunneling NS-TFET 


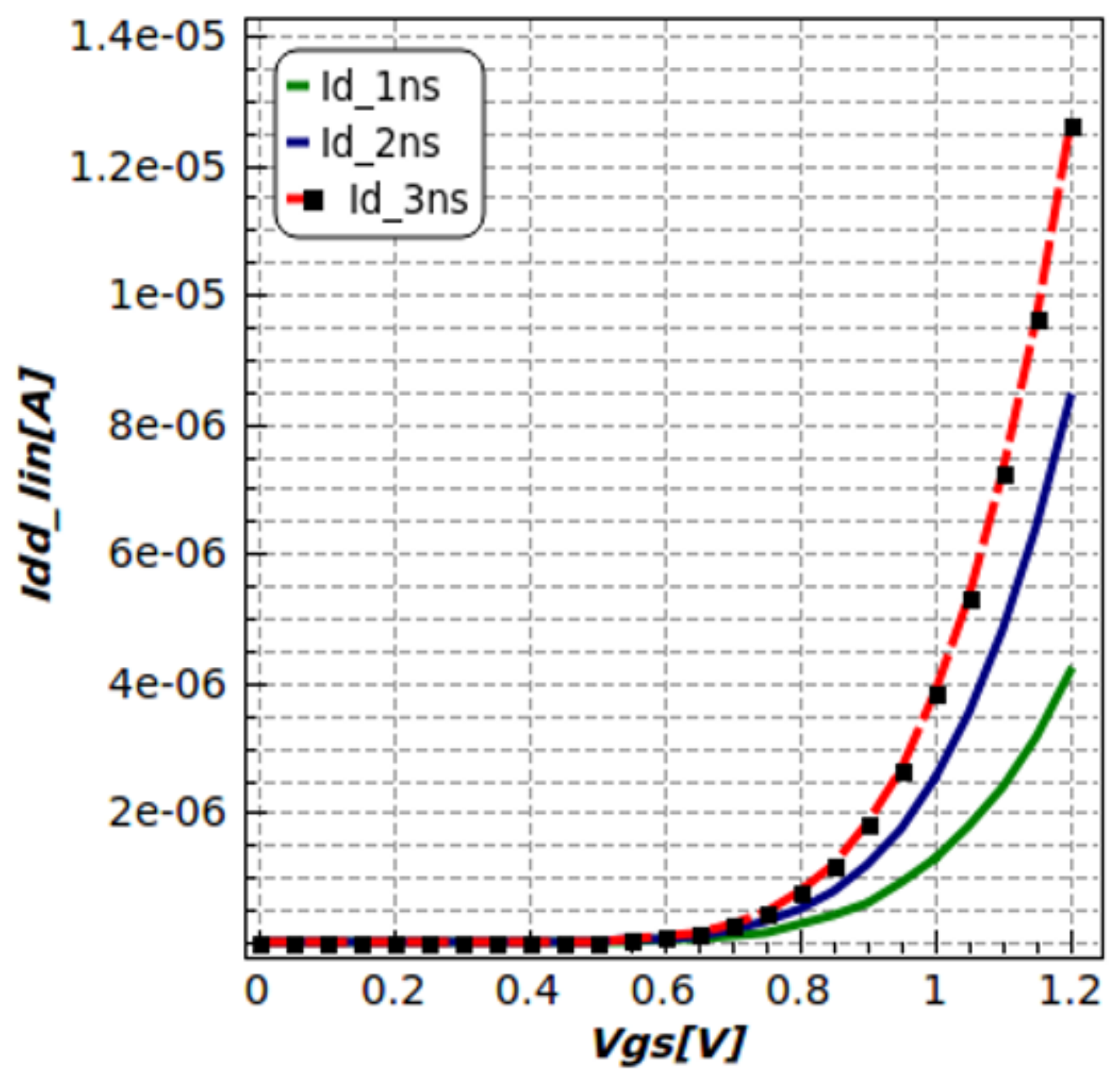

Figure 3

Simulation for single, double, and triple-stacked NS-TFET. 


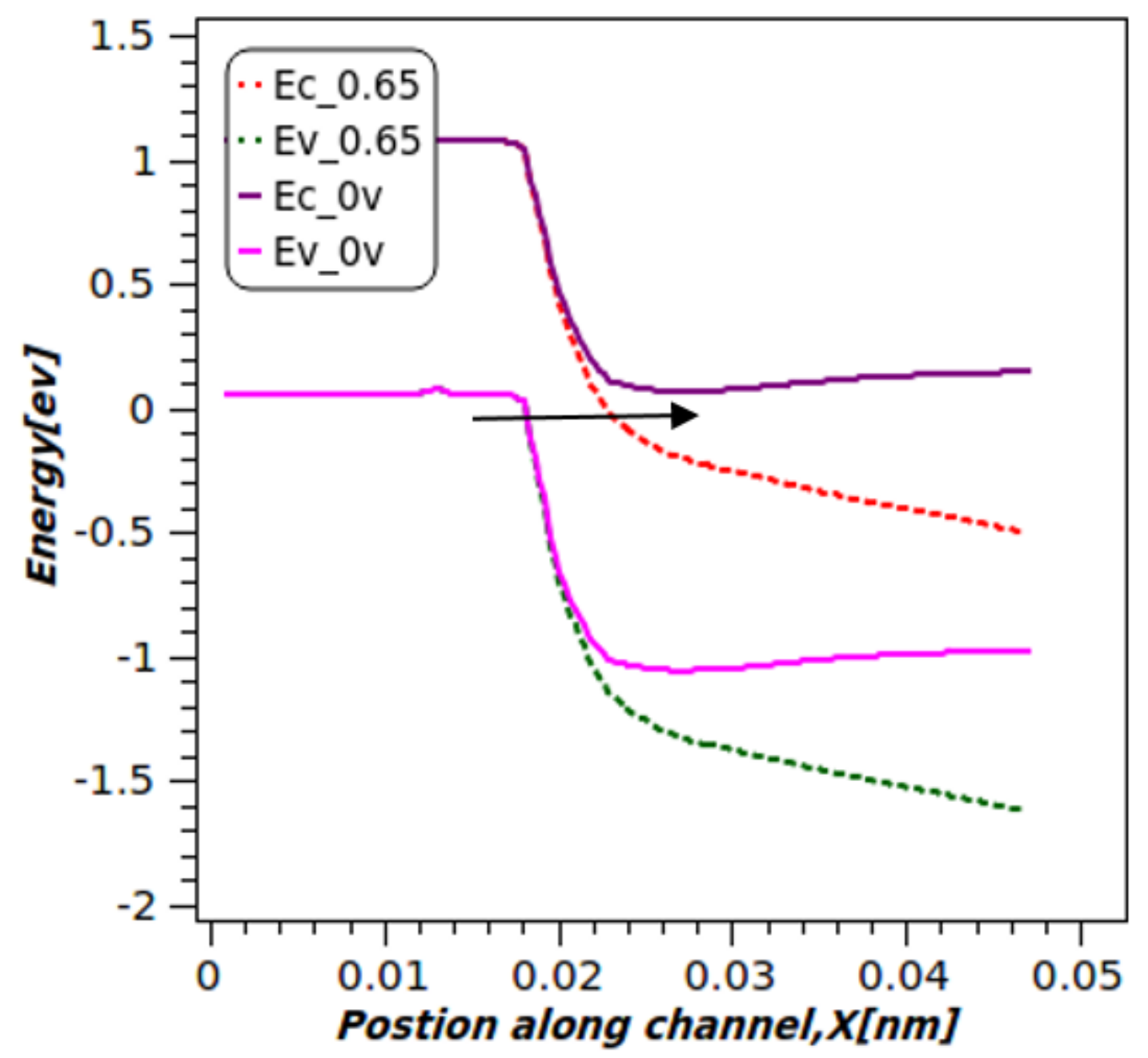

Figure 4

Band diagram of the middle layer of NS-TFET at OFF state $(\mathrm{Vgs}=0 \mathrm{~V})$ and $\mathrm{ON}$ state $(\mathrm{Vgs}>0)$ 


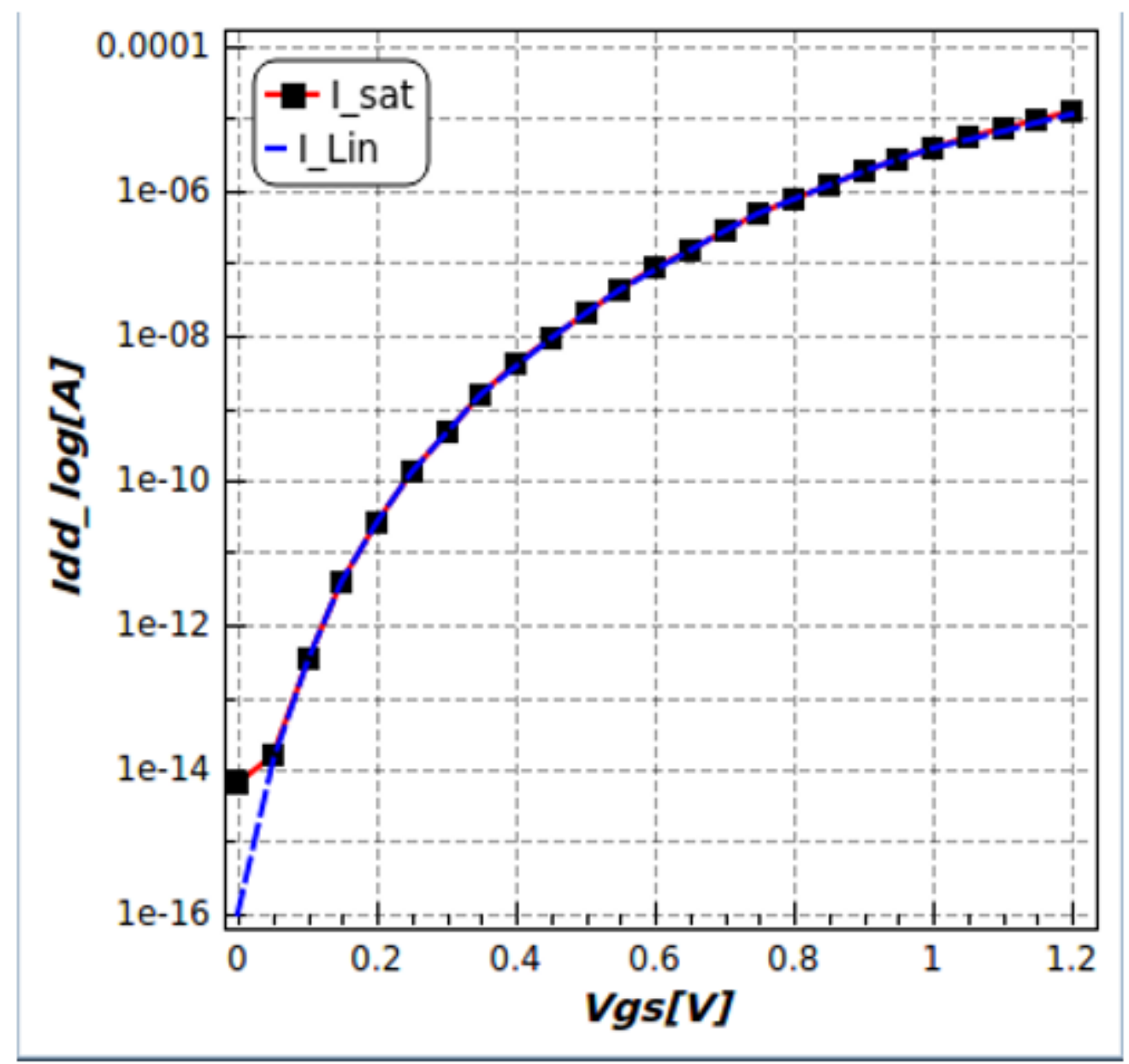

Figure 5

Transfer characteristics plot of NS-TFET at Vds $=0.10 \mathrm{~V}$ and Vds $=0.65 \mathrm{~V}$ 


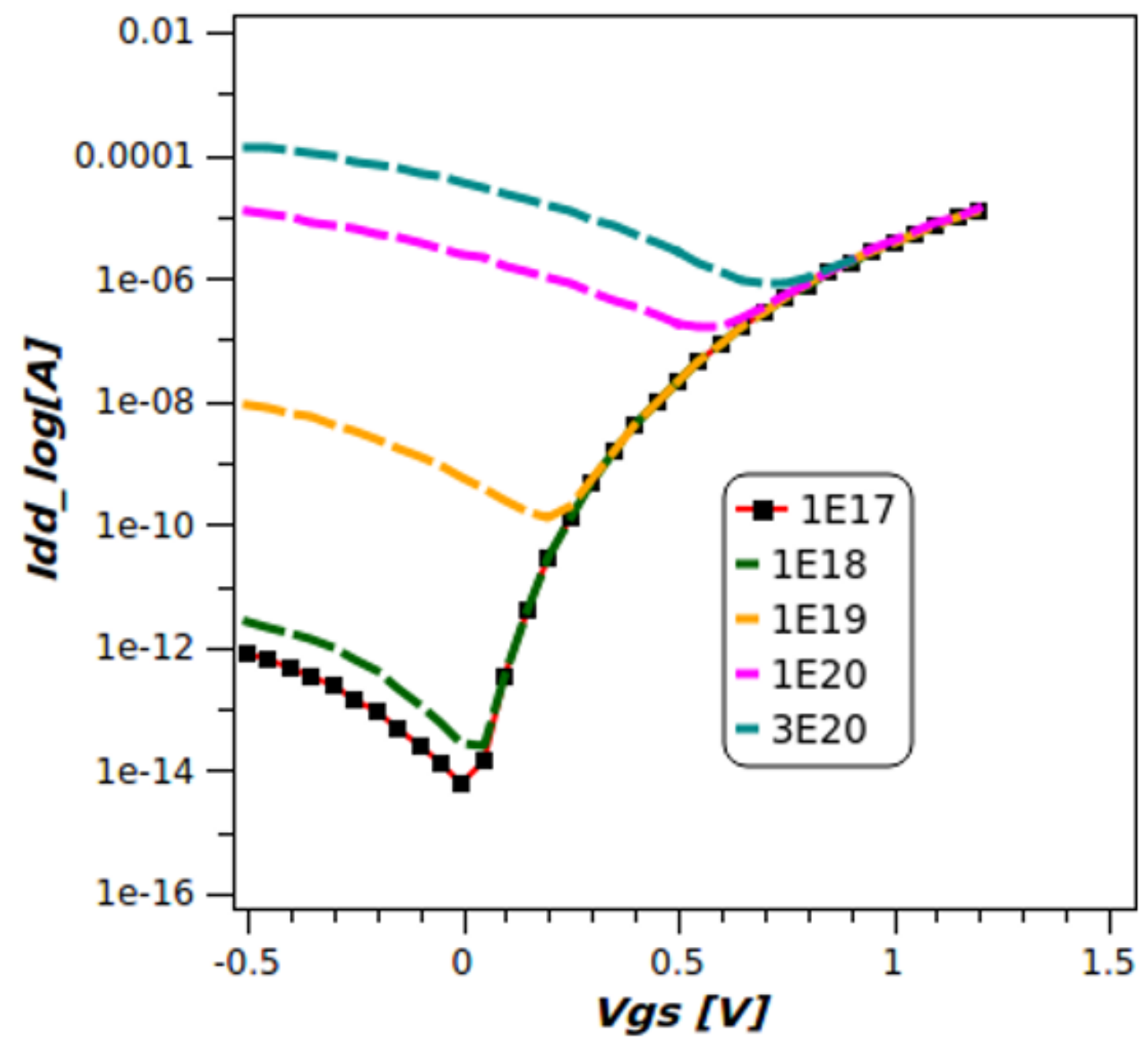

Figure 6

Transfer characteristics curve under different drain doping concentrations. 


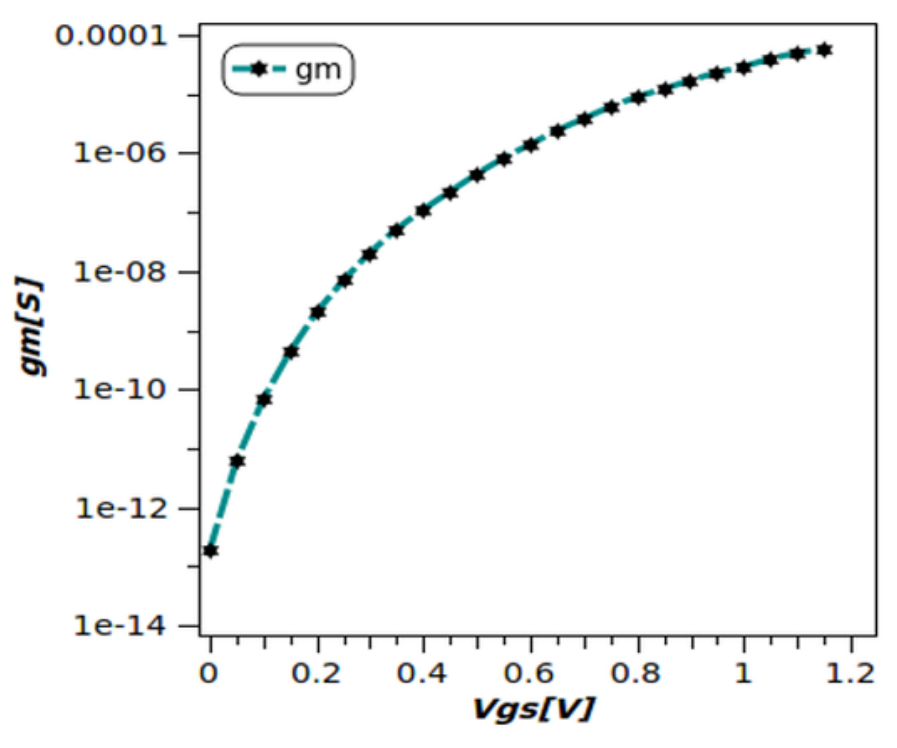

A)

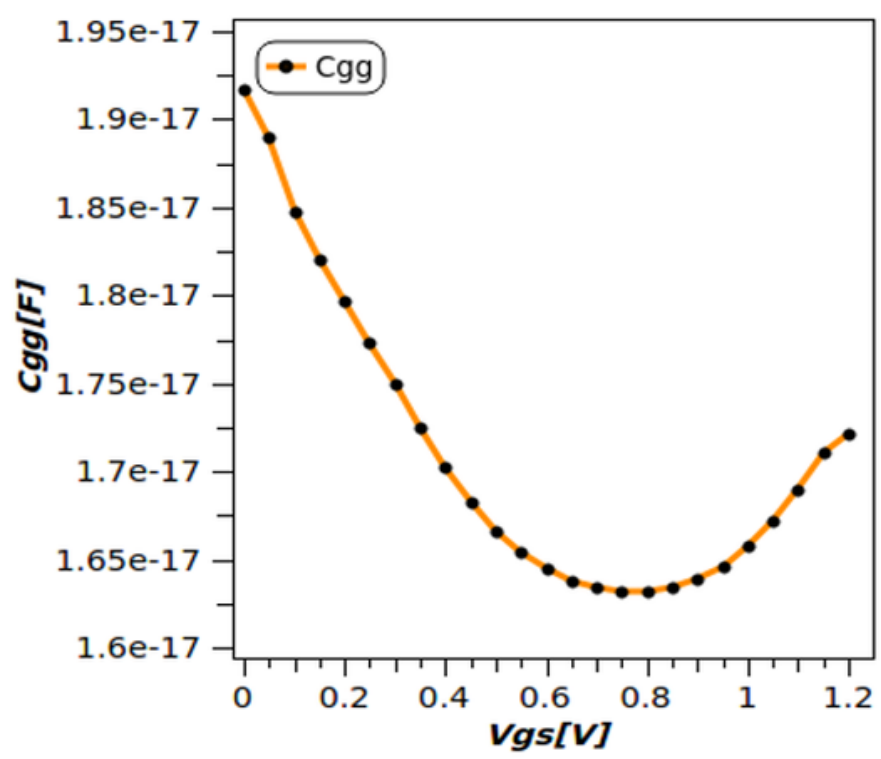

C)

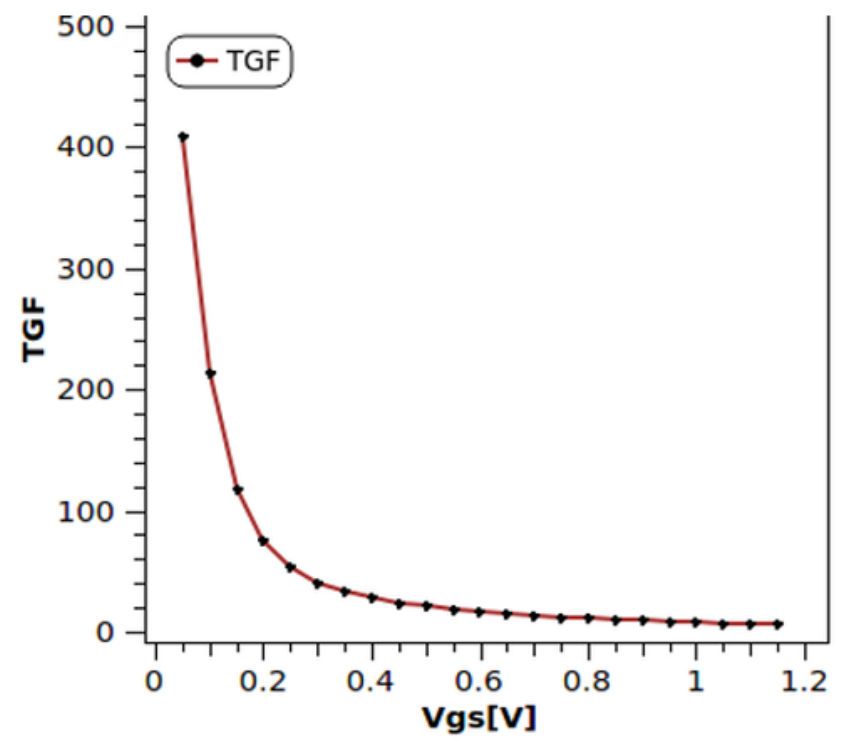

B)

\section{Figure 7}

a: Transconducatnce graph of Ns-TFET device. b: TGF plot of Ns-TFET device. c: Total gate capacitance (Cgg) plot of Ns-TFET device. 


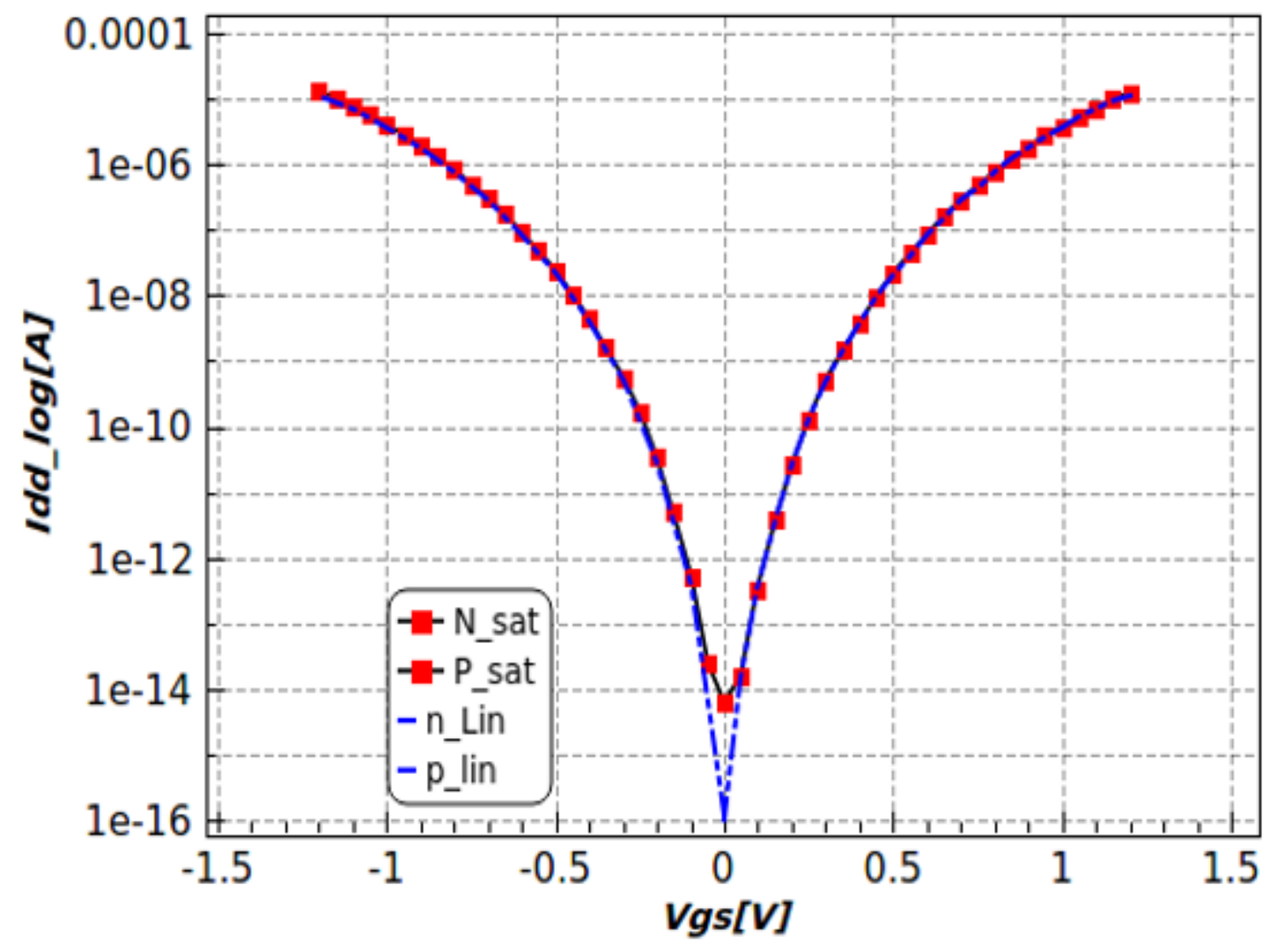

Figure 8

Transfer characteristics plot of p-NS-TFET device. 